\title{
APLICAÇÃO DOS ÍNDICES DE GEODIVERSIDADE E CLUSTERIZAÇÃO COM O USO DO K-MEANS
}

Eric Oliveira Pereira*

\section{Resumo}

O presente artigo refere-se à aplicação da metodologia de Xavier-da-Silva (2004) para a obtenção de índices de geodiversidade. Os índices de geodiversidade calculados foram os de GeodiversidadeEspecífica, Geodiversidade Múltipla e Geodiversidade Múltipla Ponderada. Este ultimo foi agrupado em 10 classes distintas com a utilização do algoritmo K-Means. O resultado final foi um mapa que representa as classes de geodiversidade para Minas Gerais. Por fim foi feita uma análise dos resultados obtidos na aplicação metodológica.

Palavras-chave:diversidade natural, geoconservação, geoprocessamento, métodos quantitativos.

\section{Abstract}

The present work intended to apply Xavier-da-Silva (2004) methodology to obtain the geodiversity index. Geodiversidade Específica, Geodiversidade Múltipla e Geodiversidade Múltipla Ponderada weretheindexcalculated.The GeodiversidadeMúltiplaPonderada.weregrupe in 10 distinct class by K-Means algorithm that represent Minas GeraisGeodiversity. In theendtheresultswereanalised.

Keywords:natural diversity, geoconservartion, geoprocessing, quantitative methods.

•Universidade Federal de Minas Gerais - UFMG. ericpereiraufmg@gmail.com 


\section{1- INTRODUÇÃO}

O conceito de geodiversidade é relativamente novo. Sua utilização seinicia a partir de 1990, consolidando-se nos últimos 10 anos desse século. Autores como Eberhard e Gray, introduzem o conceito de geodiversidade. Para Eberhard (1997) a geodiversidade consiste na diversidade natural entre os aspectos geológicos, geomorfológicos e pedológicos. Gray (2004) tem uma definição bastante parecida com a de Eberhard, contudo, além de considerar a geodiversidade como a diversidade natural entre os aspectos geológicos, geomorfológicos e pedológicos, estende sua aplicação aos estudos de planejamento territorial, mas sem se distanciar da ênfase a geoconservação.

Segundo Xavier-da-Silva (2001) o conceito de Geodiversidade, que foi submetido à comunidade ambientalista, foi definido como a variabilidade de características ambientais encontradas em uma área geográfica. Já Veiga (1999) apresenta o conceito de geodiversidade como à expressão das particularidades do meio-físico de uma determinada região geográfica, porém leva em consideração a litologia, a geomorfologia, o clima, o solo e as águas, além disso, condiciona a morfologia da paisagem e a diversidade biológica e cultural. O estudo da geodiversidade é, em sua opinião, uma ferramenta imprescindível de gestão ambiental e norteador das atividades econômicas. Estudos sobre geodiversidade têm sido aplicados para geoconservação, e para o planejamentoterritorial e envolvem a análise da interação dos fatores abióticos incluindo o clima, a relevo e a geologia, constituindo uma importante ferramenta para a gestão ambiental e norteador das atividades econômicas.

Os índices de geodiversidade podem ser calculados com os métodos que são denominados diretos e também com os indiretos. No caso da metodologia de Xavier-daSilva o cálculo é feito de maneira indireta. A opção por esse método é uma alternativa para a aplicação em grandes áreas já que o método direto exigiria um levantamento de campo de grande detalhe, o que é inviável para áreas muito grandes.

O termo "geodiversidade indireta" é utilizado para definir os cálculos de geodiversidade que levam em conta fatores que influenciam na geodiversidade, mas que não constituem uma variabilidade geológica ou geomorfológica de elementos “em si”. 
Os cálculos de variabilidade de orientação, altitude, declividade, rugosidade, paisagens, entram neste conceito. Este tipo de cálculo já havia sido realizado em escalas amplas e lugares pouco estudados: ambientes em que o trabalho de campo é impraticável, mas que necessita igualmente de informações para poder ser gerado.

"Generally, one of the limiting factors to environmental studies is the scarcityof financial resources. It is often costly or even impossible to determine anentire search focus area or areas ofstudy that will allocate more investigative effort, depending on the size of the area. To this end, it is essential to apply a methodology that satisfies these conditions in a clear, accurate and reliable, affordable and quick way" (Grigio et al. 2011).

Ainda segundo Xavier-da-Silva(2004) existem vários índices para indicação da Geodiversidade. Estes índices são:

- Geodiversidade Específica Número de classes de cada variável encontrado em um dado polígono.

- Geodiversidade de Posição Ordenação dos polígonos de acordo com a Geodiversidade de cada um dos grupos, do menos diverso ao mais diverso.

- Geodiversidade MúltiplaConsiste na soma simples de todas as classes de todas as variáveis em um mesmo polígono.

- Geodiversidade Múltipla Ponderada Consiste na divisão do índice de Geodiversidade Múltipla pela área do polígono, isso permite uma comparação entre polígonos de diferentes tamanhos.

- Geodiversidade de Posição Múltipla Ordenação dos polígonos de acordo com a Geodiversidade Múltipla Ponderada de cada um dos grupos, do menos diverso ao mais diverso.

No presente trabalho foram calculados os índices de GeodiversidadeEspecífica, Múltipla e MúltiplaPonderada. A área escolhida foi o estado de Minas Gerais. O estado possui 853 municípios, para os quais foi possível estabelecer os índices com a utilização das ferramentas de geoprocessamento e a aplicação da metodologia de Xavier-da-Silva (2004). O software ArcGis 10.1 foi utilizado para o tratamento das informações obtidas, bem como o cruzamento das mesmas. Posteriormente foi utilizado o software SPSS para o agrupamento dos índices em 10 diferentes classes. O método K-means foi aplicado para a 
obtenção destas classes de geodiversidade para o estado, por fim foi elaborado ummapa de geodiversidade para o estado de Minas Gerais.

O objetivo deste trabalho foi o de aplicar a metodologia de Xavier-da- Silva (2004) para a obtenção dos índices de Geodiversidade Específica, Múltipla e Múltipla Ponderada. Além disso, foi objetivo deste criar 10 grupos diversos de Geodiversidade para o estado com o uso do método K-Means.

Para tanto foram utilizadas as bases cartográficas referentes à vegetação, solo, geologia, declividade,topografia e relevo. Todas essas basespodem ser encontradas na base de dados "Bases de Geodiversidade Estaduais", no site doGeobank do Serviço Geológico do Brasil (CPRM). ${ }^{1}$

$\mathrm{Na}$ próxima secção do artigo estão descritas e explicadas as etapasmetodológicas para a obtenção do resultado final deste trabalho, o mapa de Geodiversidade de Minas Gerais.

\section{METODOLOGIA}

\subsection{Obtenção e tratamento das bases cartográficas}

O cálculo de Da Silva (2004), para a geodiversidade é feito de forma indireta neste tipo de cálculo não são feitos levantamentos específicos de cada uma das variáveis que compõem a geodiversidade e sim um agrupamento de fatores que influenciam na composição dessas variáveis. Seria isso um tipo cálculo que leva em consideração variáveis secundárias e, assim, é possível inferir a sobre a diversidade de um dado ambiente.

Neste caso foram utilizadas as bases referentes a geologia, declividade, topografia, solos e vegetação. Assim, as bases citadas foram extraídas do levantamento da CPRM, intitulado Mapas Estaduais de Geodiversidade.

Foi necessário desmembrar as informações que estavam agrupadas em dois arquivos shape. Para isso foi utilizado o softwareArcGis 10.1, com suas ferramentas do ArcTool Box.

Os dados referentes a relevo, topografia, declividade e geologia foram obtidas no site do Geobank. Todas essas informações estavam agrupadas em um único arquivo shapepara o estado de Minas Gerais e se referem a geodiversidade do estado.

Sendo assim, foi necessário, para a aplicação da metodologia, desassociar cada uma dessas informações em shapes únicos.

\footnotetext{
${ }^{1}$ Site para acesso ao GEOBANK/CPRM: http://geobank.sa.cprm.gov.br/ 
Para a separação foi seguido o fluxograma da figura 1, procedimento que foi repetido para cada uma das variáveis, esse mesmo fluxograma foi aplicado para a base de solos de onde foram obtidos os tipos de solos e asclasses de vegetação.



Fig. 1: Criação das shapes utilizadas para o cálculo da geodiversidade de Minas Gerais.

Com esta etapa foi possível obter cada uma das variáveis necessárias a obtenção dos índices. A próxima etapa consiste em associar cada uma das variáveis ao shape de municípios de Minas Gerais, uma vez que o cálculo terá como polígono base o limite dos municípios. O que se deseja obter é o número de classes de cada uma das variáveis existe para cada um dos 853 municípios mineiros. Para que isso fosse possível foi utilizada a ferramenta Spatialjoin contida no ArcToolBox do software ArcGis 10.1. Foi necessário executar o passo contido na figura 2 para cada uma das variáveis.

Concluído este passo, a shape de Minas Gerais terá todas as informaçõesassociadas. O que importa neste caso é número de vezes que cada uma das classes por variável ocorre no município. Ao abrir a tabela de atributos se vê o resultado presente na tabela 1 . 


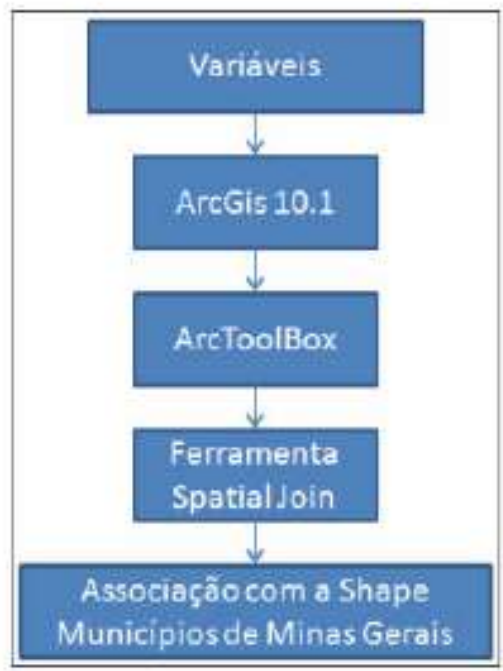

Fig. 2: Associação das variáveis a shape de municípios de Minas Gerais.

Tabela 1: Fragmento da tabela de atributos dos municípios.

\begin{tabular}{|l|r|r|r|r|r|r|r|}
\hline Municípios & Vegetação & Solo & Topografia & Declifvidadd & Geologia & Relevo \\
\hline Abadia dos Dourados & 4 & 3 & 4 & 3 & 3 & 6 \\
\hline Abaeté & 3 & 5 & 4 & 4 & 5 & 3 \\
\hline Abre Campo & 4 & 2 & 5 & 2 & 2 & 3 \\
\hline Acaiaca & 2 & 1 & 3 & 1 & 1 & 2 \\
\hline Abucena & 3 & 4 & 6 & 4 & 4 & 3 \\
\hline Agua Boa & 5 & 4 & 5 & 4 & 4 & 3 \\
\hline
\end{tabular}

A interpretação paraa tabela 1 éa seguinte: para o município de Água Boa, existem 5 classes de vegetação, 4 de solo, 5 de topografia, 4 de declividade, 4 de Geologia e 3 de Relevo. Sendo assim, já é possível perceber que a geodiversidade específica de cada um dos municípios de Minas Gerais já está calculada.

Esta etapa concluiu o tratamento das informações da base cartográfica, apróxima subsecção traz a manipulação dos dados para a obtenção dos índices de geodiversidade múltipla e múltipla ponderada.

\subsection{Cálculo da geodiversidade múltipla e múltipla ponderada}

O cálculo da geodiversidade múltipla é bastante simples, uma vez que é necessária apenas a criação de uma coluna em que a quantidadedeclasses, independente da variável, será calculada. Para tanto, é necessário criar uma coluna, que foi nomeada como Geodiversidade Múltipla. Em seguida, com o uso da ferramenta calculatefield. 
A operação para a realização docálculo é uma soma algébrica dos índices de geodiversidade específica. A figura 3 mostra a ferramenta e a fórmula utilizada para o cálculo.



Fig. 3: Field calculator com a fórmula para geodiversidade múltipla.Após a realização desta etapa a geodiversidade múltipla está calculada,como pode ser visto na coluna Geodiversidade Múltipla da tabela 2.

Tabela 2: Tabela de atributos. Geodiversidade Múltipla.

\begin{tabular}{|l|r|r|r|r|r|r|r|}
\hline & \multicolumn{9}{|c|}{ Geodiversidade Específica } & \multicolumn{2}{c|}{$\begin{array}{c}\text { Geodiversidade } \\
\text { Múltipla }\end{array}$} \\
\hline Municípios & Vegetação & Solo & Topografia & Declifvidade & Geologia & Relevo & 23 \\
\hline Abadia dos Dourados & 4 & 3 & 4 & 3 & 3 & 6 & 24 \\
\hline Abaeté & 3 & 5 & 4 & 4 & 5 & 3 & 18 \\
\hline Abre Campo & 4 & 2 & 5 & 2 & 2 & 3 & 10 \\
\hline Acaiaca & 2 & 1 & 3 & 1 & 1 & 2 & 24 \\
\hline Apucena & 3 & 4 & 6 & 4 & 4 & 3 & 25 \\
\hline Agua Boa & 5 & 4 & 5 & 4 & 4 & 3 & 13 \\
\hline Agua Comprida & 3 & 1 & 3 & 1 & 1 & 4 & 13 \\
\hline Aguanil & 2 & 2 & 3 & 2 & 2 & 2 & 33 \\
\hline Aguas Formosas & 5 & 5 & 10 & 4 & 5 & 4 & \\
\hline
\end{tabular}

Portanto a geodiversidade múltipla nada mais é que a soma de todas as classes encontradas para cada município.

Porém, esse tipo de índicedificulta a comparação entre os diversos polígonos (municípios) já que cada um deles possui uma área diferente. Para sanar esta questão o índice de geodiversidade múltipla ponderada foi aplicado. Para tal, foi necessário calcular a 
área de cada município eposteriormente aplicar uma equação simples. Neste caso optou-se por definir um índice de geodiversidade para cada 100 quilômetros quadrados dos municípios. A figura 4 mostra aferramenta utilizada para o cálculo,além disso, exibe a fórmula aplicada.



Fig. 4: Field Calculator. Geodiversidade Múltipla Ponderada.

Após a realização desta etapa a geodiversidade múltipla ponderada está calculada, como pode ser visto na coluna Geodiversidade Múltipla Ponderada da tabela 3.

Tabela 3: Tabela de atributos. Geodiversidade Múltipla Ponderada.

\begin{tabular}{|c|c|c|c|c|c|c|c|c|c|}
\hline \multicolumn{10}{|c|}{ Indices de Geodiversid ade } \\
\hline & \multicolumn{6}{|c|}{ Geodiversid ade Especifica } & & \multirow{2}{*}{$\begin{array}{c}\text { Geodiversidade } \\
\text { Múltipla }\end{array}$} & \multirow{2}{*}{$\begin{array}{c}\text { Geodiversidade } \\
\text { Múltipla Ponderada }\end{array}$} \\
\hline Municipios & Vegetaçăo & Solo & Topograflia & Declifvidada & Geologia & Relevo & Area $\mathrm{Km}^{2}$ & & \\
\hline Abadia dos Dourados & 4 & 3 & (4) & 3 & 3 & 6 & 888,738350 & 23 & 2,587938 \\
\hline Abaeté & 3 & 5 & 4 & 4 & 5 & 3 & 1818,415118 & 24. & 1,319831 \\
\hline Abre Campo & 4 & 2 & 5 & 2 & 2 & 3 & 470,360842 & 18 & 3,826849 \\
\hline Acaiaca & 2 & 1 & 3 & 1 & 1 & 2 & 100,724755 & 10 & 9,928046 \\
\hline Abucena & 3 & 4 & 6 & 4 & 4 & 3 & 834,434332 & 24 & 2,876200 \\
\hline Agua Boa & 5 & 4 & 5 & 4 & 4 & 3 & 1308,299282 & 25 & 1,910878 \\
\hline
\end{tabular}

Com isso a etapa de cálculo dos três índices de geodiversidade propostos está concluída. A dificuldade de se exibir esse resultado, de modo que se possa comparar a geodiversidade, acontece devido a grande quantidade de municípios do estado de Minas Gerais. Assim sendo, na próxima subsecção será detalhado o modo como esta questão foi solucionada. 


\subsection{Divisão do índice de geodiversidade múltipla ponderada em classes}

Com o intuito de exibir o resultado obtido na aplicação da metodologia de Xavierda-Silva (2004).Optou-se pela clusterização dos índices de geodiversidade múltipla ponderada em grupos que guardem relação entre si.

Um método não hierárquico foi escolhido para a obtenção dos grupos. Os métodos não hierárquicos possuem uma facilidade computacional. É importante saber o número de clusters que se pretende dividir a população de dados.

O K-means foi o algoritmo não hierárquico escolhido para a definição dos grupos. A equação 1 representa este algoritmo.

$$
J=\sum_{j=1}^{k} \sum_{i=1}^{n}\left\|x_{i}^{(j)}-c_{j}\right\|_{\text {equação } 1 .}^{2}
$$

De onde || $\mathrm{Xi}(\mathrm{j})-\mathrm{Cj}|| 2$ é responsável pelo cálculo da distância média entre um ponto dado Xi (j)e o centro do cluster $\mathrm{Cj}$. Contudo a aplicação do algoritmo não significa encontrar os melhores grupos. Para isso são executadas várias iterações a fim de melhorar o resultado final.

Sendo assim, os dados foram extraídos do softwareArcGis 10.1 no formato .dbf, com isso foi facilmente importado para o software SPSS.

O SPSS já possui esse algoritmo implementado, o que facilitou a aplicação desta etapa do trabalho. Para isso basta indicar o número de grupos a ser formado, o número de iterações e a variável a ser agrupada. A figura 5 exibe a tela de configuração para a aplicação do K-means. 


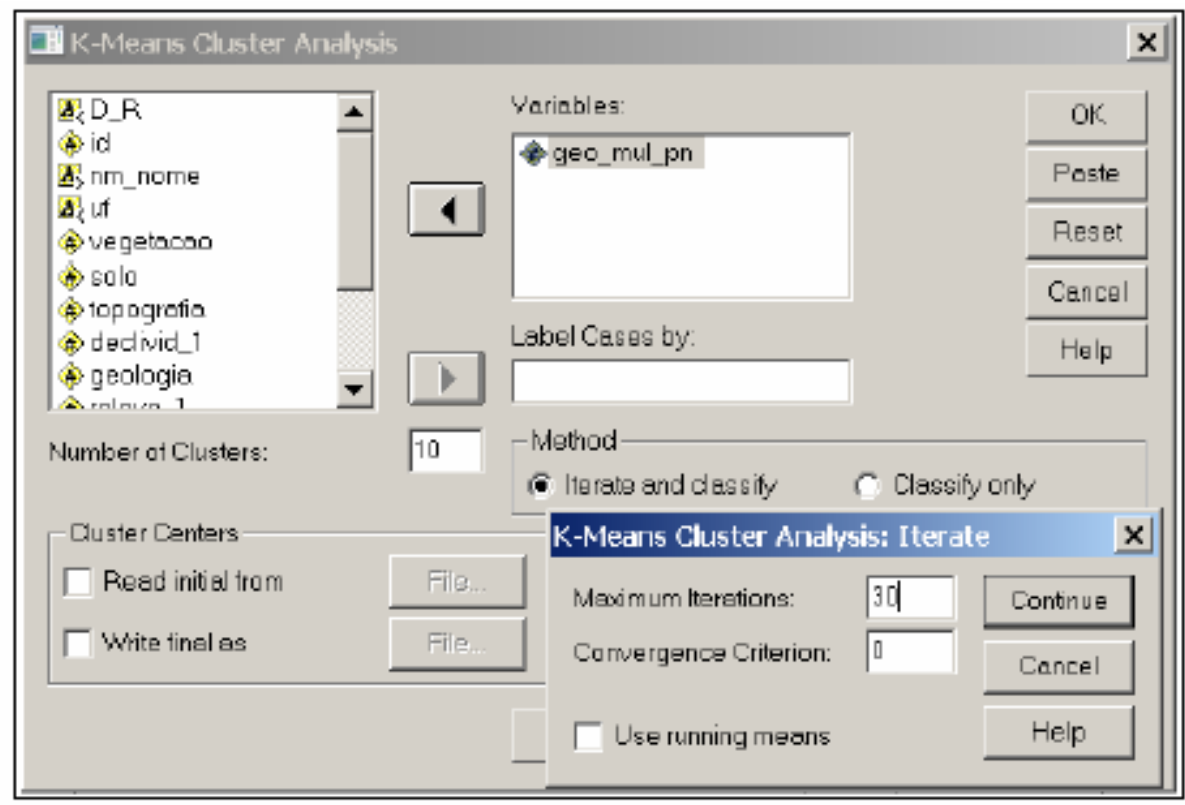

Fig. 5: K-Means Cluster Anlysis. SPSS.

Neste trabalho foi indicada variável geodiversidade múltipla ponderada para a clusterização, com 10 clusters distintos e com um máximo de 30 iterações. Sendo assim, foram formados os grupos e os dados foram transferidos novamente para o softwaresArcGis 10.1 para a elaboração do mapafinal.

\section{RESULTADOS E DISCUSSÕES}

Como resultado deste trabalho tem-se os cálculos de geodiversidade específica, múltipla e múltipla ponderada. Estes dados podem servisualizados de forma tabular, noentanto, devido ao grande número de municípios do estado torna-se maçante a análise de todos eles. A tabela 4 é um fragmento desse resultado para que se possa ter uma noção da organização dos dados. 
Tabela 4: Fragmento do Resultado final do cálculo dos índices de geodiversidade

\begin{tabular}{|c|c|c|c|c|c|c|c|c|c|}
\hline \multicolumn{10}{|c|}{ Indices de Geodiversidade } \\
\hline & \multicolumn{6}{|c|}{ Geodiversidade Especifica } & & \multirow{2}{*}{$\begin{array}{c}\text { Geodiversidade } \\
\text { Múltipla }\end{array}$} & \multirow{2}{*}{$\begin{array}{c}\text { Geodiversidade } \\
\text { Múltipla Ponderada }\end{array}$} \\
\hline Municípios & Vegetação & Solo & Topografia & Declifvidade & Geologia & Relevo & Area $\mathrm{Km}^{2}$ & & \\
\hline Abadia dos Dourados & 4 & 3 & 4 & 3 & 3 & 6 & 888,738350 & 23 & 2,587938 \\
\hline Abaeté & 3 & 5 & 4 & 4 & 5 & 3 & 1818,415118 & 24. & 1,319831 \\
\hline Abre Campo & 4 & 2 & 5 & 2 & 2 & 3 & 470,360842 & 18 & 3,826849 \\
\hline Acaiaca & 2 & 1 & 3 & 1 & 1 & 2 & 100,724755 & 10 & 9,928046 \\
\hline Apucena & 3 & 4 & 6 & 4 & 4 & 3 & 834,434332 & 24 & 2,876200 \\
\hline Agua Boa & 5 & 4 & 5 & 4 & 4 & 3 & 1308,299282 & 25 & 1,910878 \\
\hline Agua Comprida & 3 & 1 & 3 & 1 & 1 & 4 & 510,374371 & 13 & 2,547150 \\
\hline Aguanil & 2 & 2 & 3 & 2 & 2 & 2 & 235,642039 & 13 & 5,516842 \\
\hline Aguas formosas & 5 & 5 & 10 & 4 & 5 & 4 & 814,532852 & 33 & 4,051402 \\
\hline Aguas Vermelhas & 6 & 4 & 7 & 4 & 4 & 3 & 1270,324644 & 28 & 2,204161 \\
\hline Aimorés & 7 & 7 & 14 & 7 & 6 & 6 & 1331,955351 & 47 & 3,528647 \\
\hline Aluruoca & 9 & 4 & 8 & 4 & 4 & 1 & 653,942484 & 30 & 4,587559 \\
\hline Alagoa & 5 & 1 & 3 & 1 & 1 & 1 & 163,264340 & 12 & 7,350043 \\
\hline Albertina & 3 & 2 & 2 & 2 & 1 & 3 & 55,994282 & 13 & 23,216656 \\
\hline Além ParaÝba & 4. & 3 & 4 & 3 & 3 & 3 & 501,174199 & 20 & 3,990628 \\
\hline Alfenas & 6 & 2 & 9 & 2 & 2 & 3 & 846,497303 & 24 & 2,835213 \\
\hline Alfredo Vasconcelos & 2 & 3 & 2 & 3 & 3 & 1 & 126,660103 & 14 & 11,053204 \\
\hline Almenara & 14 & 7 & 11 & 6 & 7 & 7 & 2305,209062 & 52 & 2,255761 \\
\hline Alpercata & 3 & 3 & 4 & 3 & 3 & 4 & 171,581700 & 20 & 11,656255 \\
\hline Alpinópolis & 9 & 2 & 6 & 2 & 2 & 7 & 455,023483 & 28 & 6,153529 \\
\hline
\end{tabular}

Na tabela é possível observar os três índices calculados para o estado de Minas Gerais. Essa tabela exibe todas as variáveis utilizadas bem como o nome e área de cada um dos municípios. Esses mesmo dados poderiam compor um mapa em que cada um dos municípios assumiria um valor discreto diferente. Mas, mais uma vez seria algo que nãopossibilitaria uma análise significativa, uma vez que observar 853 valores distintos é inviável.

Para viabilizar a elaboração de uma tabela síntese bem como de um mapa optou-se pela criação dos clusters. Estes clusters foram elaborados com o uso do software SPSS. O método utilizado foi o K-Means. Este método permitiu agrupar os municípios a partirdos índices de geodiversidade múltipla. A escolha de 10 classes diferentes de forma aleatória, não houve critério para que a escolha fosse feita. A tabela 5 , elaborada a partir dos dados da tabela 4 acrescidos do número dos grupos obtidos no SPSS foi sintetizada no Excel e mostra o número de municípios por grupo. 
Tabela 4: Municípios por Grupo.

\begin{tabular}{|c|r|}
\hline Grupos & Quantidade de Municípios \\
\hline 1 & 256 \\
\hline 2 & 222 \\
\hline 3 & 8 \\
\hline 4 & 2 \\
\hline 5 & 1 \\
\hline 6 & 117 \\
\hline 7 & 1 \\
\hline 8 & 10 \\
\hline 9 & 44 \\
\hline 10 & 192 \\
\hline Total geral & $\mathbf{8 5 3}$ \\
\hline
\end{tabular}

O método aplicado,desenvolvido por Xavier-da-Silva, possui três resultados distintos. O primeiro deles é a Geodiversidade Específica, que consiste no número de classes de uma variável em um mesmo município. Este índice é bastante simples e não traz muitas informações sobre a variedade de uma dada área, apenas indica a de uma única variável. Sua observação isolada não leva a muitas conclusões, uma vez que se observa apenas, por exemplo, o quanto a vegetação ou a geologia variam em um município, e o propósito dos índices de geodiversidade é ter uma interpretação sobre a variabilidade com a integração das variáveis ambientais.

O outro resultado da análise é o Índice de Geodiversidade Múltipla. Esse índice consiste na soma de toda a geodiversidade específica de uma dada área. Assim, o índice de Geodiversidade Múltipla é importante para uma análise integrada das variáveis ambientais, uma vez que são consideradas todas asvariáveis utilizadas no trabalho. Porém, esse índice acarreta em um problema grave. A comparação entre os polígonos que representam áreas distintas é errônea, já que áreas grandes com o um número grande classes pode não ser mais geodiverso que uma área muito pequena com poucas classes.

Para sanar o problema da Geodiversidade Múltipla, é possível padronizar os valores dos índices calculados. Para isso basta acrescentar a informação da área e fazer uma operação algébrica simples. Assim, o último dos índices é obtido. O Índice de Geodiversidade Múltipla Ponderada é, portanto, a aplicação da equação 2, onde o Índice de Geodiversidade Múltipla é multiplicado pela área do município e o resultado é posteriormente dividido por 100. Isso indica que o índice obtido refere-se a Geodiversidade para cada $100 \mathrm{Km}^{2}$ do município. 


\section{[Geodiversidade Múltipla] \\ [AREA]}

equação 2 .

A tabela 4 é o resultado da aplicação dos três tipos de índices de geodiversidade propostos por Xavierda-Silva 2004. Desta tabela partiu-se para a criação das classes de geodiversidade para o estado de Minas Gerais. Dentre os índices calculados o de Geodiversidade Múltipla Ponderada parece ser o mais coerente para uma representação do que vem a ser a geodiversidade dos municípios de Minas Gerais. Sendo assim o Índice de Geodiversidade Múltipla foi escolhido para a elaboração do mapa de Geodiversidade de Minas Gerais.

Como a inserção de 853 valoresdistintos, um para cada município, é inviável e resultaria em um mapa de difícil interpretação, optou-se pela divisão em 10 classes de geodiversidade distintas.

Para isso foi utilizado o software SPSS, que permitiu a aplicação do algoritmo KMeans para a obtenção de 10 grupos distintos. O método K-Means é interessante, pois é capaz de realizar os agrupamentos, levando em consideração a distância média das observações em relação ao centroide de modo rápido, e com 30 iterações retorna grupos satisfatórios. O modelo não hierárquico, que é o caso do K-Means, é responsável pela velocidade de processamento dos dados. Nesta etapa forma inseridos os Índices de Geodiversidade Múltipla Ponderada no software para que fossem calculados os grupos.

O resultado final do trabalho foi a elaboração de um mapa de Geodiversidade de Minas Gerais baseado no agrupamento realizado pela aplicação do K-Means a partir dos Índices de Geodiversidade Múltipla Ponderada. O mapa ficou bastante claro e indicar bem a variação da geodiversidade no estado. Contudo algumas observações podem ser feitas em relação ao resultado. O mapa está representado na figura 6 . 


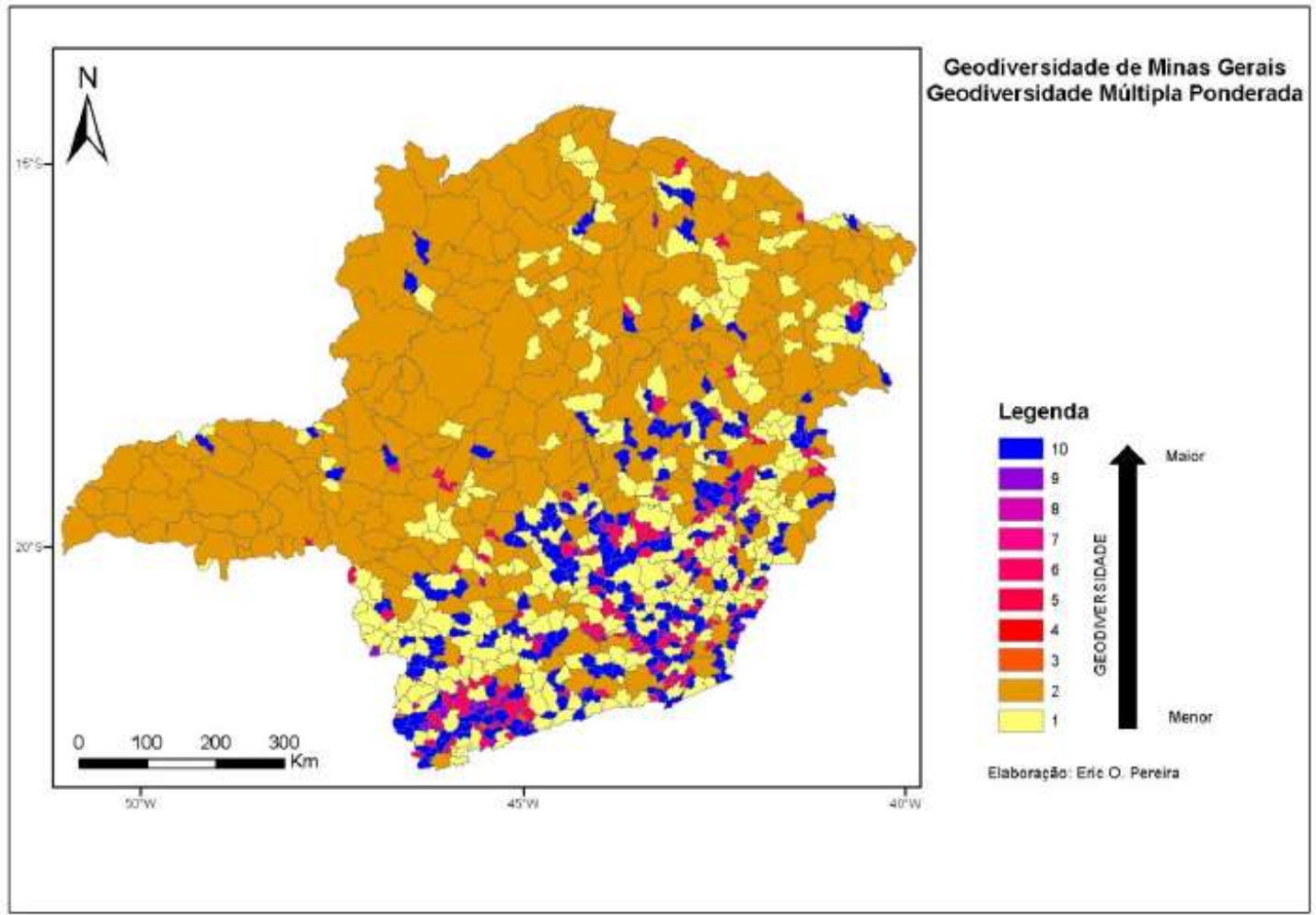

Fig. 6: Mapa de Geodiversidade de Minas Gerais.

Ao observar o mapa da figura 6 percebe-se uma homogeneidade em grande porção do oeste e noroeste mineiro em relação a geodiversidade. Esses municípios foram agrupados no grupo 2, que tem uma geodiversidade relativamente baixa. Já os municípios da porção sul, sudeste e alguns da região central, bem como outros aleatoriamente distribuídos no estadoficaram no grupo 5 e 10 o que indica, respectivamente, uma geodiversidade média e alta.

Essa diferença no grau de geodiversidade entre os municípios pode ter sido acarretada por diversos motivos. Um deles, o que é o ideal, é a possibilidade de os ambientes naturais, reais, serem coincidentes com o mapeamento. As regiões oeste e noroeste do estado seriam realmente menos diversas, com menor variabilidade ambiental aquelas localizadas na porção sul e sudeste do estado. Essa seria a possiblidade ideal e que daria maior credibilidade a aplicação da metodologia de Xavier-da-Silva 2004. Porém outras possiblidades foram levantadas com base no resultado tais como a qualidade das bases cartográficas, a escala de mapeamento e aplicação metodológica. Essas questões estão expostas na próxima secção. 


\section{CONCLUSÃO}

$\mathrm{O}$ trabalho realizado queresultou neste artigo foi bastante interessante. $\mathrm{O}$ objetivo geral de se aplicar a metodologia de Xavier-da- Silva e obter grupos de geodiversidade com o algoritmo K-Means foi atendido. O resultado final foi o mapa de Geodiversidade do Estado de Minas Gerais apresentado na secção anterior.

Parte-se do pressuposto que osíndices encontrados representam a realidade da diversidade natural dos fatores abióticos da área de estudo. Porém existem algumas considerações que devem ser feitas acerca do resultado obtido e também da aplicação metodológica.

No que diz respeito às basescartográficas, por exemplo, não se sabe sobre a qualidade das informações levantadas para uma ou outra porção do estado. Existe a possiblidade de terem sido compiladas informações de escalas diferentes de levantamento, apesar das informações estarem agrupadas em único arquivo shape. As porções sul e sudeste podem ter um maior grau de detalhamento, com uma escala maior e as outras regiões podem ter escala

relativamente menor. Isso influenciaria diretamente o resultado da aplicação da metodologia que pressupõe que toda a área tenha a mesma escala de mapeamento.

Outra observação válida éinteressante observar que os índices foram calculados com referência à divisão político-administrativa. Sabe-se que não é o ideal a aplicação desta forma. Neste trabalho está divisão foiutilizada na tentativa de se aplicar e ter meios de comparar os índices encontrados para o estado mineiro. O ideal seria ter como base de comparação algum tipo de divisão baseados nas características naturais da área de estudo, como compartimentação geomorfológica, bacias hidrográficas, entre outros.

Contudo, o trabalho foi válido, pois os objetivos propostos com a aplicação da metodologia de Xavier-da- Silva (2004) foram atingidos e ainda a utilização do método KMeans na criação das classes de geodiversidade do estado foi possível.

\section{BIBLIOGRAFIA}

CÂMARA, Gilberto. Representação Computacional de dados geográficos.In: CÂMARA, Gilberto. In: Casanova, Marco; Davis, Clodoveu; Vinhas, Lúbia; Queiroz, Gilberto Ribeiro de; Câmara, Gilberto. Banco de Dados Geográficos. Curitiba: MundoGEO.2005. 
EBERHARD, R. (Ed.). Pattern and process: towards a regional approach to national estate assessment of geodiversity. Technical Series, n. 2.Australian Heritage Commission; Environement Forest Taskforce, Environment Australia, Caberra, 1997.

GEOBANK CPRM<http://geobank.sa.cprm.gov.br/>Acesso: 30Novembro 2012.

GRAY, M. Geodiversity: valuing and conserving abiotic nature. New York: John Wiley \& Sons, 2004. 434 p.

GrigiO, A. M.; AMARO, V. E.; DIODATO, M. A, and CASTRO, A. F. de, 2011. Determination of indices ofmultiple and Multiple WeightedGeodiversity of landscape of the Piranhas-Assuriver, Rio Grande do Norte - Brazil. Journal of Coastal Research, SI 64 (Proceedings of the 11th International Coastal Symposium), 1668 - 167. Szczecin, Poland, ISSN 0749-0208.

VEIGA, A. T. C. A geodiversidade e o uso dos recursos minerais da Amazônia. Terra das Águas, Brasília: NEAz/UnB, n. 1, p. 88-102, 1999.

XAVIER-DA-SILVA, J. Geoprocessamento para análiseambiental. Rio de Janeiro: Ed. Jorge Xavier da Silva, 2001. 228 p.

XAVIER-DA-SILVA, J. et al. Índices de geodiversidade: aplicações de SGI em estudos de biodiversidade. In: GARAY, I.; DIAS, B. F. S. (Orgs.). Conservação da biodiversidade em ecossistemas tropicais: avanços conceituais e revisão novas metodologias de avaliação e monitoramento. Rio de Janeiro: Vozes, 2001. p. 299-316.

XAVIER DA SILVA, J. ; ZAIDAN, Ricardo Tavares. Geoprocessamento e análise ambiental: aplicações. Rio de Janeiro: Bertrand Brasil, 2004. 363 p.

XAVIER-DA-SILVA, J. \& CARVALHO FILHO, L. M. Índice de geodiversidade da Restinga da Marambaia (RJ), um exmplo do geoprocessamento aplicado a geografia física. Revista de Geografia, v. 17, n. 1, 2001. P. 57-64. 